\title{
Energy Efficient Clustering in Sensor Networks with Mobile Agents
}

\author{
Mahdi Lotfinezhad and Ben Liang \\ Department of Electrical and Computer Engineering, University of Toronto, Ontario, M5S 3G4 \\ Email:\{mlotfinezhad, liang\}@ comm.utoronto.ca
}

\begin{abstract}
Wireless sensor networks with mobile access points are effective tools to collect data in a variety of environments. Low-cost and low-power sensors in the reachback operation contend for the channel to transmit their own data packets to the mobile agent. This data communication should be designed to ensure energy efficiency and low latency. In this paper, we propose a clustering scheme for wireless sensor networks with reachback mobile agents (C-SENMA) toward that goal. C-SENMA groups sensors into clusters such that nodes communicate only with the nearest clusterhead $(\mathrm{CH})$ and the $\mathrm{CH}$ takes the task of data aggregation and communication with the mobile agent. In our scheme, CHs use a low-overhead medium access control (MAC) mechanism very similar to the conventional ALOHA to contend for the channel. Using results from random geometry theory, we analyze the clustering performance under the realistic MAC algorithm. Our analysis enables us to obtain the optimal average cluster size which minimizes energy consumption. We justify our analysis results by extensive simulations according to various clustering parameters. Furthermore, we study the effect of underlying physical layer characteristics on the amount of energy reduction achievable by the proposed clustering architecture.
\end{abstract}

\section{INTRODUCTION}

There has been extensive research in wireless sensor networks with flat and clustered ad hoc architectures [1][2]. Traditionally in such networks, sensors are limited in terms of energy supply, processing power, storage, and are aimed to be low-cost and low-power devices [3][2]. Because ordinary sensors are typically battery operated, energy efficient protocol design is one of the challenging issues in wireless sensor networks [2].

Recently, sensor networks with mobile agents, SENMA, have been proposed in [3][4]. In contrast to ordinary sensors, mobile agents are powerful hardware units with sophisticated transceivers. They have much less constraint on their communication capabilities as well as their processing power. Mobile agents visit the network either periodically or when the application requires them to gather data or perform network maintenance. Manned/unmanned aerial vehicles are examples of such agents [3]. It has been shown that the simple topology of SENMA reduces energy consumption and improves the scalability of sensor networks [3].

The availability of a mobile agent makes direct communication between ordinary sensors and the mobile agent possible. This opens up many interesting problems in medium access control [4][5]. However, there has not been any detailed study to analyze the effect of clustering in SENMA. In this work, we propose and analyze a novel clustering scheme for SENMA

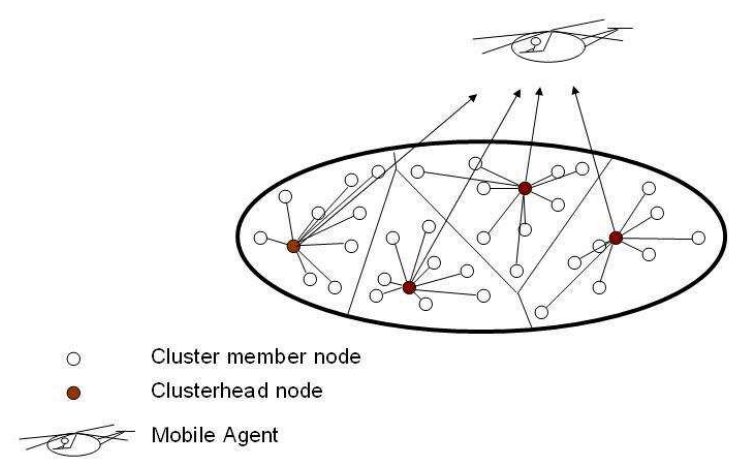

Fig. 1. Architecture of a typical network in C-SENMA

(C-SENMA) where stationary nodes form clusters and only the clusterhead $(\mathrm{CH})$ has the task of communicating with the mobile agent. C-SENMA generates clusters periodically in a random manner using any general clustering algorithm, for example the one used in [1]. CHs receive data packets from their cluster-members and perform data fusion. Then, they transmit the compressed data directly back to the mobile agent as shown in Fig.1. Thanks to the simple topology of C-SENMA, CHs can use a simple MAC protocol to contend for the reachback channel. We show that data aggregation along with clustering in C-SENMA reduces energy consumption up to $90 \%$ in typical scenarios. Furthermore, we obtain the optimal probability of being a $\mathrm{CH}$ (or equivalently the optimal average cluster size) to minimize energy consumption while maintaining an acceptable level of (data collection) latency. In contrast to previous works on clustering algorithms which assume a contention and error free MAC [6], our cross-layer approach takes into account the effects of MAC on the clustering performance. To the best of out knowledge, this is the first work in the area of mobile agents which analytically investigates the performance of clustering under realistic MAC assumptions.

The rest of this paper is organized as follows. In Section II, we describe the related work in detail. In Section III, we explain the underlying reachback channel model. Details about our protocol are provided in Section IV. In Section V, we evaluate the performance of the proposed protocol. Then, we present our numerical and simulation results in Section VI. Finally, we conclude the paper in Section VII.

\section{RELATED WORK}

Recently, various issues have been investigated in SENMA including QoS information retrieval [5] and source reconstruc- 
tion [7]. While these problems may seem rather different, all of them are highly coupled with medium access control. Although many MAC protocols have been proposed for conventional flat and clustered ad hoc sensor networks [8], they may not be suitable for the mobile agent scenario. In traditional communication networks, packet arrival is random while this may not be true in SENMA. In fact, if a sensor is functioning properly, it has a packet to send when the mobile agent visits the network [5]. This fact along with the energy efficiency motivates designing new MAC protocols for SENMA.

In [4], nodes use opportunistic ALOHA (O-ALOHA) to access the channel in SENMA without clustering. In our work, we assume a much simpler MAC to show how clustering enhances the network performance. In [5], a different scheme is proposed where the mobile agent in each time slot notifies a group of nodes to transmit in the next timeslot. Although this scheme achieves high throughput, it requires that most nodes listen to the channel most of the time. In addition, [5] does not consider the effect of clustering. In our scheme, each node contends for the channel independently of the other nodes and without direct scheduling by the mobile agent.

On the other hand, many clustering protocols have been proposed for wireless sensor networks [1][6][9]. All of these protocols assume that data sinks are stationary. Specifically in [6], hierarchical clustering is proposed, and optimal clustering parameters are obtained to minimize the total energy consumption. However, they assume that the underlying MAC is contention and error free. In addition, they do not consider a mobile data sink.

In this work, using the same algorithm as in [6] to generate clusters, we consider a cross-layer approach by which we are able to consider the effect of the MAC protocol on clustering performance in the mobile agent scenario.

\section{Reachback Channel Model}

We assume that the mobile agent has multi-packet reception (MPR) capability and assume that time is slotted. In [10], a general model for channels with MPR capability is developed which is also used in our model. The channel is characterized by $r_{n k}$, the probability of $k$ successful receptions when a total of $n$ packets are transmitted in a timeslot. We can summarize the MPR property of the channel by the following stochastic matrix, $\mathbf{R}$, whose elements are all possible values for $r_{n k}$.

$$
\mathbf{R}=\left(\begin{array}{cccccc}
r_{10} & r_{11} & 0 & 0 & 0 & \ldots \\
r_{20} & r_{21} & r_{22} & 0 & 0 & \ldots \\
r_{30} & r_{31} & r_{32} & r_{33} & 0 & \ldots \\
\vdots & \vdots & \vdots & \vdots & \vdots & \ddots
\end{array}\right)
$$

Using the above model, we can obtain the expected number of correctly received packets when $n$ packets are transmitted as

$$
C_{n}=\sum_{k=1}^{n} k r_{n k} .
$$

To determine the entries of $\mathbf{R}$, we need to consider the physical layer specifications. We use the same method taken in [11] to obtain $r_{n k}$ and assume that CDMA is used to access the channel. For the sake of completeness, we briefly describe how to obtain $r_{n k}$. Let $S, \sigma^{2}$, and $n+1$ denote the spreading gain, the power of additive white Gaussian noise, and the total number of users respectively. Under the assumption that the multiaccess interference from other users is Gaussian, the biterror-rate (BER) is

$$
p_{e}(n)=Q\left(\sqrt{\frac{3 S}{n+3 S \sigma^{2}}}\right),
$$

where $Q($.$) denotes the complementary error function [12]. If$ we assume that errors occur independently in a packet with $N_{b}$ bits and up to $t$ errors in a packet can be corrected by a block error control code, then the probability of successfully demodulating a packet is

$$
p_{s}(n)=\sum_{j=0}^{t} B\left(j, N_{b}, p_{e}(n)\right) .
$$

where $B\left(j, N_{b}, p_{e}(n)\right)$ denotes the probability mass at the value $j$ of a Binomial random variable with total $N_{b}$ trials and the success probability $p_{e}(n)$. Consequently, when $n$ nodes are transmitting the probability of $k$ successful receptions is

$$
r_{n k}=B\left(k, n, p_{s}(n-1)\right) .
$$

\section{PRotocol Description}

We assume that $N_{t}$ sensors are randomly deployed over a remote area $A$ (e.g., a desert or a forest) and sensors periodically sense the environmental parameters (e.g., temperature) and record these information. For the sake of analysis, we assume that the area $A$ is a circle with radius $R$. To gather the sensed data, a mobile agent, e.g., an airplane, periodically visits the network and initiates a round of data collection. In this operation, both the amount of time and energy needed to collect data are of our main concern. When the mobile agent visits the network, it broadcasts a beacon which initiates communication, network maintenance, and (if required) cluster formation. In this paper, we assume that sensors directly communicate with the $\mathrm{CHs}$ in one hop.

In C-SENMA, cluster formation is triggered by the mobile agent. To ensure that the load of being a $\mathrm{CH}$ is rotated among all nodes, the cluster formation is performed every multiple rounds of data collection. To construct clusters, each node selects itself as a $\mathrm{CH}$ with fixed probability $p_{c}$. Later, we show how this probability affects the protocol performance. If a node becomes a $\mathrm{CH}$, it broadcasts an advertisement packet (ADV) to announce its status as well as the PN code that should be used by its cluster-members for data communication. Based on the received signal strength of ADV packets, each node approximates its distance to the nearby $\mathrm{CHs}$ and joins the cluster of the nearest $\mathrm{CH}$.

After reception of the beacon from the mobile agent, communication in the clusters starts. We assume that nodes within a cluster use TDMA to access the channel using the same PN code broadcasted by the their $\mathrm{CH}$. To become synchronized, nodes use the beacon broadcasted by the mobile agent. 
When all data is received by a $\mathrm{CH}$, it performs data aggregation and contends for the reachback channel to forward the data up to the mobile agent. In this work, we assume that ideal aggregation is possible, i.e., an arbitrary number of packets can be compressed down into one packet [1][6][13]. Examples of such aggregation include finding maximum, minimum, or average of the collected information. In C-SENMA, CHs use CDMA to communicate with the mobile agent via the MPR physical interface (of the mobile agent).

Our MAC scheme is slightly different from other conventional models, e.g., ALOHA. While in conventional models, traffic arrival is a continuous random process, in our scenario every $\mathrm{CH}$ has a packet to send, and after this packet is received correctly, the $\mathrm{CH}$ does not have any other packets to send until the next round of data collection.

In C-SENMA, after a $\mathrm{CH}$ finishes data collection, it transmits the aggregated data with probability $p_{N}^{t}$ to the mobile agent, where $N$ represents the number of contending $\mathrm{CHs}$ in the current timeslot. If this packet is not received correctly, then the $\mathrm{CH}$ retransmits the packet with a new probability in the next timeslot (depending on the updated number of contending $\mathrm{CHs}$ ). To utilize the MPR channel capability and maximize the throughput, we need to find the optimal probability of transmission, $p_{N}^{t}$. Therefore, we have

$$
p_{N}^{t}=\arg \max _{p} \sum_{n=1}^{N} B(n, N, p) C_{n} .
$$

We mentioned earlier that the cluster formation is triggered by the mobile agent. Therefore, the mobile agent has the status of all $\mathrm{CHs}$ (by receiving ADV packets broadcasted by each $\mathrm{CH})$. This mechanism gives the necessary information about the number of clusters. On the other hand, based on the correctly demodulated data, the mobile agent can update the number of remaining clusters. This updated number should be fed back to the $\mathrm{CHs}$ and is used as $\mathrm{N}$ by $\mathrm{CHs}$ to find the corresponding $p_{N}^{t}$. As well as the updated information, the feedback contains acknowledgements of correctly received packets.

\section{PRotocol AnAlysis}

If clusters are of equal size, then they ideally finish their TDMA schedule at the same time and try to contend for the channel at the same next timeslot. This means that the number of remaining $\mathrm{CHs}$, whose packets are not received by the mobile agent, is equal to the number of contending CHs. In reality, because clusters are formed randomly, they have different sizes. Consequently, their corresponding $\mathrm{CHs}$ finish their TDMA schedule and start to contend for the channel at different timeslots. As a result, the number of remaining clusters (which is the available information based on which $\mathrm{CHs}$ choose the $p_{N}^{t}$ ) is not necessarily equal to the number of contending $\mathrm{CHs}$ in a given time slot. We base our analysis on equal size clusters. Later in Section VI, we show that our analysis predicts the total energy consumption very well when clusters are formed randomly according to our protocol.

\section{A. Markov Chain Analysis}

It can be easily seen that the number of contending $\mathrm{CHs}$ forms a finite discrete Markov chain. Consider the transition from the state $(m)$ to the state $(n)$ and let $t_{m n}$ denote the probability of that transition. Conditioned on $i \mathrm{CHs}$ transmitting in the next timeslot, $m-n$ packets should be received (by the mobile agent) correctly to produce such a transition. Therefore, considering all possibilities we have

$$
t_{m n}=\left\{\begin{array}{ll}
\sum_{i=m-n}^{m} B\left(i, m, p_{m}^{t}\right) r_{i, m-n} & n \leq m, m \neq 0 \\
0 & n>m \\
1 & n, m=0
\end{array} .\right.
$$

Clearly, there is one absorbing state, (0), in the Markov chain. Given that the current state is $(m)$, we can calculate the mean time to absorbtion, $a_{m}$, and mean number of transmissions, $u_{m}$, until absorbtion. Let

$$
\begin{aligned}
& \mathbf{a} \triangleq\left[a_{1}, \ldots, a_{N_{t}}\right], \\
& \mathbf{u} \triangleq\left[u_{1}, \ldots, u_{N_{t}}\right] .
\end{aligned}
$$

Defining $\mathbf{W}$ as the transition matrix of all transient states, we have [14]

$$
\mathbf{a}=(\mathbf{I}-\mathbf{W})^{-1} \mathbf{1},
$$

where $\mathbf{I}$ is the identity matrix and $\mathbf{1}$ represents a vector with all entries equal to 1 . To compute $\mathbf{u}$, we assume that the current state of the chain is $N$. In this case, $n$ packets are transmitted with probability $B\left(n, N, p_{N}^{t}\right)$, and the current state will transit to the state $N-i$ with probability $r_{n i}$. Considering all possibilities for $n$, we have

$$
\begin{aligned}
u_{N} & =\sum_{n=0}^{N} B\left(n, N, p_{N}^{t}\right) n \\
& +\sum_{n=0}^{N} \sum_{i=0, i \neq N}^{n} B\left(n, N, p_{N}^{t}\right) r_{n i} u_{N-i} \\
& =N p_{N}^{t}+\sum_{K=1}^{N} t_{N K} u_{K} .
\end{aligned}
$$

Summarizing the above equation for all values of $N$ (all states) in a vector format, we get

$$
\begin{aligned}
& \mathbf{u}=\mathbf{v}+\mathbf{W u}, \\
& \mathbf{u}=(\mathbf{I}-\mathbf{W})^{-1} \mathbf{v},
\end{aligned}
$$

where, $\mathbf{v}=\left[1 p_{1}^{t}, \ldots, i p_{i}^{t}, \ldots, N_{t} p_{N_{t}}^{t}\right]$ is a vector representative of the average number of transmissions given that the current state is $i$.

Assuming that each node selects itself as a $\mathrm{CH}$ independently of other nodes, the actual number of $\mathrm{CHs}$ in the network has a Binomial distribution and so does the initial condition of the Markov chain:

$$
P\left(N_{0}=n\right)=B\left(n, N_{t}, p_{c}\right) .
$$


We can obtain the expected number of transmissions $E(T r)$ and timeslots $E(T s)$ until absorbtion by

$$
\begin{aligned}
& E[T r]=B\left(0, N_{t}, p_{c}\right) \mathbf{u}\left(N_{t}\right)+\sum_{n=1}^{N_{t}} B\left(n, N_{t}, p_{c}\right) \mathbf{u}(n),(7) \\
& E[T s]=B\left(0, N_{t}, p_{c}\right) \mathbf{a}\left(N_{t}\right)+\sum_{n=1}^{N_{t}} B\left(n, N_{t}, p_{c}\right) \mathbf{a}(n) .(8)
\end{aligned}
$$

The first terms in the above formulas come form the fact that if there is no $\mathrm{CH}$ in the network, then all nodes send directly to the mobile agent.

\section{B. Energy Consumption Analysis}

Our goal here is to obtain the expected value of the total energy consumption, $E[U]$, needed to gather data within clusters and transmit data to the mobile agent.

Let $E_{D P}$ be the energy required to process a bit for data aggregation purposes and $E\left(N_{0}\right)=N_{t} p_{c}$ represent the average number of clusters. We assume that if a node wants to transmit a packet to another node at a distance $d$, then it should consume $c_{0}+c_{1} d^{\alpha}$ units of energy. Based on this energy model, let $E\left(S_{c_{0}+c_{1}\left|x_{i}\right|^{\alpha}}\right)$ denote the expected value of the total energy consumed by all cluster-members (within a cluster) to communicate with their $\mathrm{CH}$. In $E\left(S_{c_{0}+c_{1}\left|x_{i}\right|^{\alpha}}\right),\left|x_{i}\right|$ is the distance between the $i$ th cluster-member and its $\mathrm{CH}$. Based on these definitions, we have

$$
\begin{aligned}
E[U]= & N_{b}\left\{E[\operatorname{Tr}]\left(c_{0}+c_{1} H^{\beta}\right)\right. \\
& \left.+E\left(N_{0}\right) E\left(S_{c_{0}+c_{1}\left|x_{i}\right|^{\alpha}}\right)+N_{t} E_{D P}\right\},
\end{aligned}
$$

where $H$ is the height at which the mobile agent flies over the network and, $\alpha$ and $\beta$ show the roll-off factors for the two types of data communication, i.e., within clusters and from $\mathrm{CHs}$ to the mobile agent. We assume that $H$ is large enough so that the distance between all nodes and the mobile agent can be approximated by $H$. In (9), the first term in braces shows the energy required to communicate with the mobile agent. The second term represents within cluster energy consumption and the last one shows the processing energy expenditure. To calculate (9), we need to compute $E\left(S_{c_{0}+c_{1}\left|x_{i}\right|^{\alpha}}\right)$. This expectation is computed using random geometry theory [15] which is also used in [6].

Based on our assumptions, nodes are uniformly distributed over the area $A$. This point process can be approximated by a Poisson point random process whose density is $\lambda=\frac{N_{t}}{A}$. If every node is going to be a $\mathrm{CH}$ with probability $p_{c}$, then it can be shown that the $\mathrm{CH}$ point process is also a Poisson process with density $\lambda_{1}=p_{c} \lambda$ and the remaining points constitute another Poisson process with density $\lambda_{0}=\left(1-p_{c}\right) \lambda$ [14].

Furthermore, in our cluster formation we have assumed that every node joins the cluster of the nearest $\mathrm{CH}$. Therefore, the area $A$ is tessellated to a set of Voronoi cells. Let $\mathcal{C}_{0}$ represent a typical Voronoi cell whose nucleus is located at the origin, $\Pi_{0}$ denote the Poisson point process associated with the non- $\mathrm{CH}$ nodes, and $x_{i}$ be a member of $\Pi_{0}$. We define a function $f\left(x_{i}\right)$ as a property of $x_{i}$, e.g., its distance to the $\mathrm{CH}$, and $S_{f}$ as the summation of that property over all cluster-members, i.e.,

$$
S_{f}=\sum_{x_{i} \in \prod_{\mathbf{o}}} f\left(x_{i}\right) \mathbf{1}\left\{x_{i} \in \mathcal{C}_{0}\right\}
$$

where $\mathbf{1}\{$.$\} is the indicator function. From [15] we can compute$ the expected value of $S_{f}, E\left(S_{f}\right)$, as follows

$$
E\left(S_{f}\right)=\lambda_{0} \int f(x) \exp \left(-\lambda_{1} \pi|x|^{2}\right) d x
$$

In our case, $f\left(x_{i}\right)$ is given by our energy consumption model, which is $c_{0}+c_{1}\left|x_{i}\right|^{\alpha}$. When $\alpha$ is 4 , plugging this form of $f\left(x_{i}\right)$ into (10) leads to

$$
\begin{aligned}
E\left(S_{c_{0}+c_{1}\left|x_{i}\right|^{\alpha}}\right) & =\lambda_{0} \int f(x) \exp \left(-\lambda_{1} \pi|x|^{2}\right) d x \\
& \left.\simeq \frac{-2 \pi \lambda_{0} c_{0}}{2 a_{0}} \exp ^{-a_{0} r^{2}}\right|_{0} ^{R} \\
& +\left.2 \pi \lambda_{0} c_{1}\left(\frac{-r^{4}}{2 a_{0}}-\frac{r^{2}}{a_{0}^{2}}-\frac{1}{a_{0}^{3}}\right) \exp ^{-a_{0} r^{2}}\right|_{0} ^{R}
\end{aligned}
$$

where $a_{0}=\lambda_{1} \pi$. The approximation in (11) is due to the fact that the area $A$ is finite.

\section{Latency Analysis}

In this work, we obtain an approximate upper bound for the total latency of the data collection. It is clear that the total latency has two components. The first one is the latency introduced by the TDMA schedules within clusters. The second component is the latency introduced by the contention in the reachback channel (to the mobile agent). TDMA latency is bounded by the largest cluster size in the network. We assume a maximum cluster size that is three standard deviations above the mean. Let $n_{c}$ be the number of nodes in a cluster. For mean and variance of $n_{c}$ we have [15]

$$
\begin{aligned}
E\left(n_{c}\right) & =p_{c}^{-1}, \\
\sigma_{n_{c}}^{2} & =\frac{\lambda_{0}}{\lambda_{1}}+0.28{\frac{\lambda_{0}}{\lambda_{1}}}^{2} .
\end{aligned}
$$

On the other hand, the latency of the reachback channel is maximized when all clusters have equal sizes, i.e., when all $\mathrm{CHs}$ contend for the channel at the same time. Therefore, $E(T s)$ is the upper bound of the reachback latency. Let $L^{u}$ denote our approximate upper bound of latency. Considering the TDMA and the reachback latency bounds, we get

$$
L^{u}=E\left(n_{c}\right)+3 \sigma_{n_{c}}+E(T s),
$$

where $E(T s), E\left(n_{c}\right)$, and $\sigma_{n_{c}}$ are derived in (8), (12), and (13) respectively.

\section{Numerical Results AND Simulation}

In this section we provide our numerical results based on (9) and (14). We justify our analysis by simulating CSENMA in a wide range of parameters. Each simulation data point is obtained by averaging over 200 random realizations. 


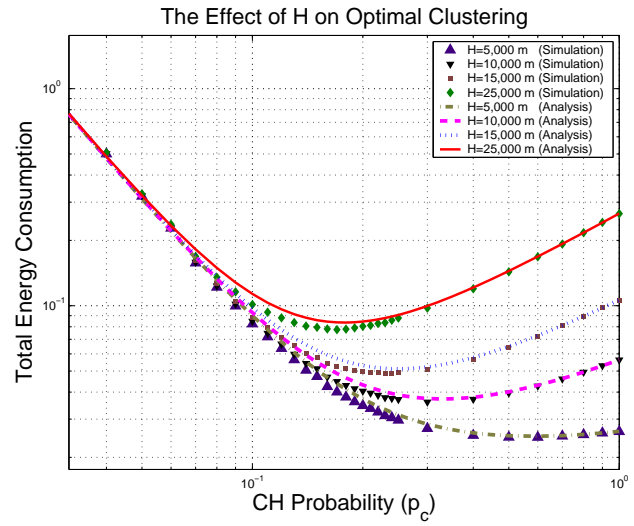

Fig. 2. Energy consumption vs. $\mathrm{CH}$ probability. Here $\beta=2$.

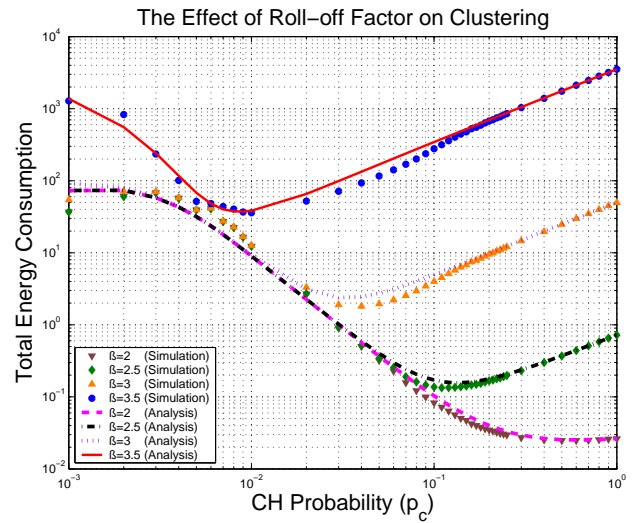

Fig. 3. The effect of roll-off factor on clustering performance. Here $H=5000$ $\mathrm{m}$.

Throughout these results, $N_{t}=1000, c_{0}=50 \mathrm{~nJ} / \mathrm{bit}[1]$, $c_{1}=0.0013 \mathrm{pJ} / \mathrm{bit} / m^{4}[1], E_{D P}=5 \mathrm{~nJ} / \mathrm{bit} / \mathrm{signal}$ [1], and we set $N_{b}=200$ bits. We choose the network radius, $R$, and the spreading gain, $\mathrm{S}$, to be 1000 meters and 32 respectively, unless otherwise stated.

Through Fig.2, Fig.3, and Fig.4, we have presented both the analysis and simulation results according to various clustering parameters. These figures suggest that for high values of $p_{c}$, there is a perfect match between our analysis and the simulation. When $p_{c}$ decreases, we expect to observe a mismatch due to the finite size of the network. In fact, when $p_{c}$ is less than 0.01 , there are only about 10 clusters on the average. Consequently, the likelihood of observing a typical cluster (with respect to a network with infinite area) decreases. Therefore, our analysis may not be accurate when $p_{c}$ is very low. On the other hand, as explained in Section V, unequal cluster sizes can affect energy consumption of the network. This effect is more pronounced at small values of $p_{c}$.

Fig. 2 shows the energy consumption of the protocol vs $\mathrm{CH}$ probability, $p_{c}$, when $\beta=2$. As can be seen, as $H$ increases the energy reduction of the clustering also increases (from $6 \%$ to more than $65 \%$ ) and the optimal $p_{c}$ decreases. This means that larger clusters lead to a better performance in terms of energy consumption. This figure also indicates that

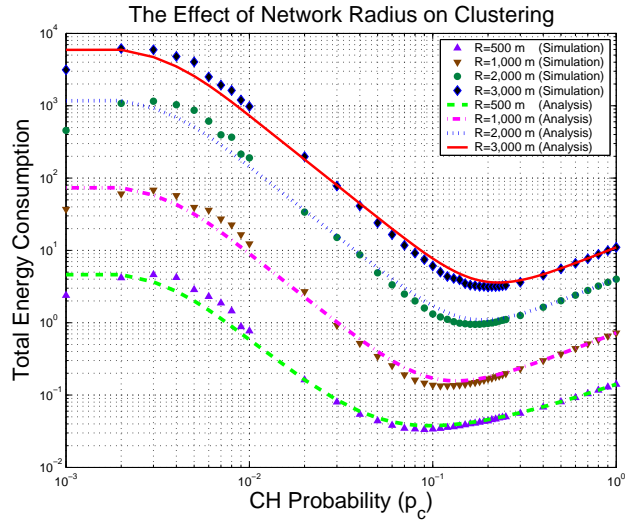

Fig. 4. The effect of network radius on clustering performance. Here $\beta=2.5$ and $H=5 R$.

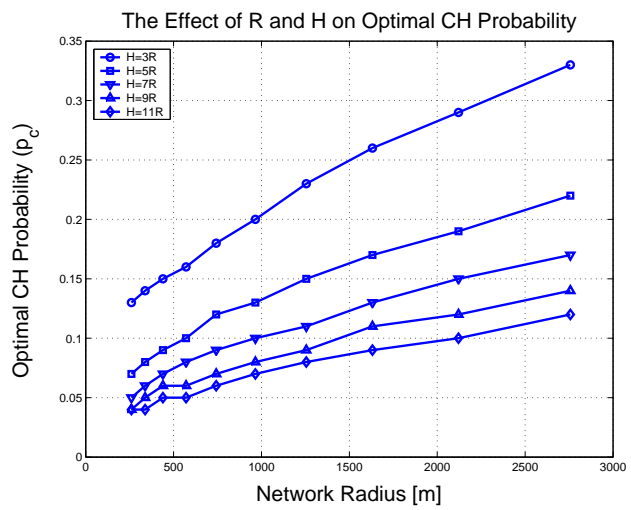

Fig. 5. The effect of network radius on optimal $\mathrm{CH}$ probabilities. Here $\beta=$ 2.5 .

at a low $p_{c}$, the performance is weakly coupled with $H$. In fact, as $\mathrm{CH}$ probability decreases, larger clusters are formed and the average distance to the $\mathrm{CHs}$ increases. Consequently, the energy consumption within clusters (to communicate with $\mathrm{CHs}$ ) dominates the energy consumed to communicate with the mobile agent.

In Fig.3, we have demonstrated the effect of roll-off factor on the optimal clustering. As expected, increasing roll-off factor translates to more energy expenditure needed to communicate with the mobile agent. Therefore in such cases, larger clusters are more efficient (lower $p_{c}$ ). On the other hand, it can be seen that the performance is highly sensitive to the changes in the roll-off factor and the total energy consumption is reduced more than an order of magnitudes when roll-off factor takes values equal or larger than 3.

Fig.4 shows the effect of network radius on clustering performance while the total number of nodes is kept constant at 1000 . We set $\beta=2.5$ to be more conservative in our results. In addition, we choose $H=5 R$. Since a smaller network radius results in smaller distances between nodes, decreasing network radius improves clustering performance and larger clusters are optimal. Therefore, as $R$ decreases, the optimal $p_{c}$ decreases, too. Fig.5 shows the optimal probabilities as a function of network radius with the same parameters as in 


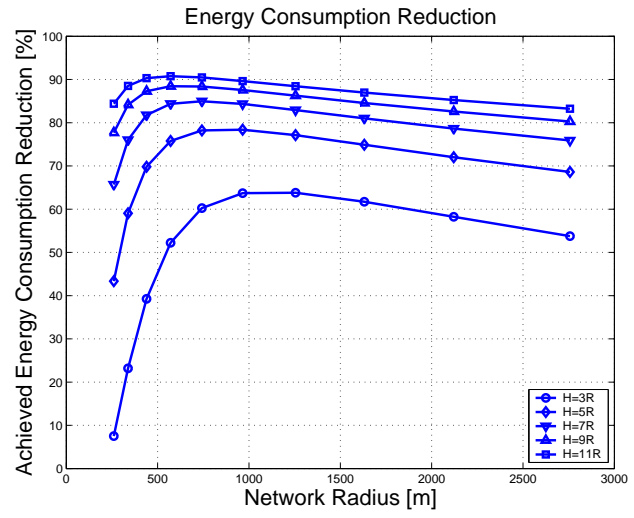

Fig. 6. The amount of energy gains vs. network radius. Here $\beta=2.5$.

Fig.4 except that we vary $H$ from $3 R$ to $11 R$. It can be observed that the increases in $R$ and $H$ increase the optimal $p_{c}$. The corresponding amount of energy reductions for various values of $R$ and $H$ is depicted in Fig.6. It can be inferred that clustering performs much better in moderately large networks.

Fig.7 shows the total latency of data collection and $L^{u}$ for different values of spreading gain $S$. Based on our model, $R$ and $H$ only can change the propagation delay which is negligible compared to the queuing delay. Therefore, results of this figure are approximately independent of $H, R$, and $\beta$. From this figure it is clear that $L^{u}$ is a tight bound for most values of $p_{c}$. Furthermore, our results indicate that increasing spreading gain directly influences the total delay. Specifically, for large values of $\mathrm{CH}$ probability, doubling $S$ halves the associated latency. When $\mathrm{CH}$ probabilities are low, few clusters are formed. This indicates that communication is mainly done within clusters. Therefore, the MPR capability of the mobile agent can not be fully utilized, and the delay to gather data within clusters (TDMA delay) dominates the delay to communicate with the mobile agent. Consequently, increasing $S$ does not decrease the system latency for small values of $p_{c}$.

Finally, combining the results from Fig.5 and Fig.7, we can conclude that the optimal clustering based on energy consumption also leads to near minimal total latency, which is reduced significantly from the case where clustering is not used, i.e., when $p_{c}=0$ or $p_{c}=1$.

\section{CONCLUSION}

In this paper, we have proposed a new clustering scheme in wireless sensor networks with mobile agents (C-SENMA). Initiated by the mobile agent, clusters in C-SENMA are formed in a distributed and random manner. We have proposed a simple MAC protocol and optimized its parameters to maximize the throughput. Based on this MAC scheme, we have obtained the optimal parameter values to make the clustering algorithm energy efficient. It has been concluded that for moderate network sizes and typical signal path loss, network energy expenditure can be reduced more than $80 \%$. We have also studied the effect of clustering on the data collection latency. We observed that the optimal cluster size based on energy consumption also leads to near minimal total latency.

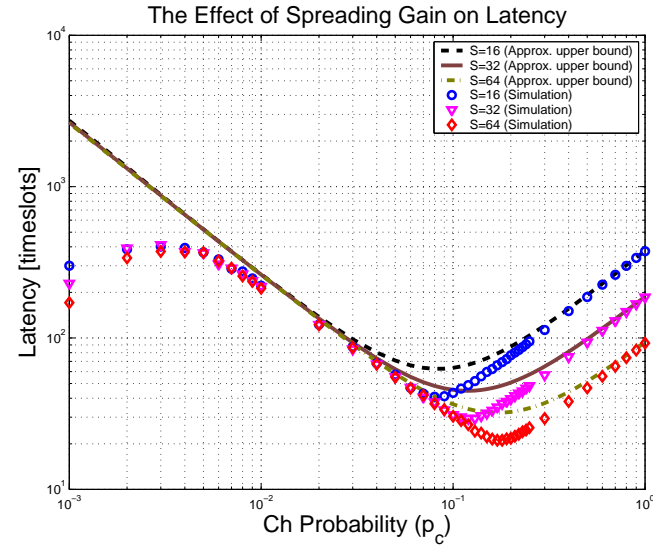

Fig. 7. The effect of spreading gain on system latency.

\section{REFERENCES}

[1] W. B. Heinzelman, A. P. Chandrakasan, and H. Balakrishnan, "An application-specific protocol architecture for wireless microsensor network," IEEE Trans. on Wireless Communications, vol. 1, pp. 660-670, Oct 2002.

[2] I. F. Akyildiz, W. Su, Y. Sankarasubramaniam, and E. Cayirci, "A survey on sensor networks," IEEE communications magazine, vol. 40, pp. 102114, Aug 2002.

[3] L. Tong, Q. Zhao, and S. Adireddy, "Sensor networks with mobile agents," in Proc. IEEE MILCOM'03, Oct. 2003.

[4] P. Venkitasubramaniam, S. Adireddy, and L. Tong, "Sensor networks with mobile access: Optimal random access and coding," IEEE Journal on Selected Areas in Communications: Special Issue on Sensor Networks, vol. 22, no. 6, pp. 1058-1068, Agu. 2004.

[5] Q. Zhao and L. Tong, "Quality-of-service specific information retrieval for densely deployed sensor networks," in Proc. IEEE MILCOM'03, Oct. 2003, pp. 591-596.

[6] S. Bandyopadhyay and E. J. Coyle, "An energy efficient hierarchical clustering algorithm for wireless sensor networks," in Proc. IEEE INFOCOM, vol. 3, March/April 2003, pp. 1713-23.

[7] M. Dong, L. Tong, and B. Sadler, "Source reconstruction via mobile agents in sensor networks: Throughput-distortion characteristics," in Proc. IEEE MILCOM, vol. 1, Oct. 2003, pp. 694-698.

[8] K. Sohrabi, J. Gao, V. Ailawadhi, and G. J. Pottie, "Protocols for selforganization of a wireless sensor network," in IEEE Personal Communications, vol. 7, Oct. 2000, pp. 16-27.

[9] B. Liang and Z. Haas, "Virtual backbone generation and maintenance in ad hoc network mobility management," in Proc. IEEE INFOCOM, March 2000, pp. 1293-1302.

[10] S. Ghez, S. Verdu, and S. Schwartz, "Stability properties of slotted aloha with multipacket reception capability," IEEE Transactions on Automatic Control, vol. 33, no. 7, pp. 640-649, July 1988.

[11] Q. Zhao and L. Tong, "A dynamic queue protocol for multiaccess wireless networks with multipacket reception," to appear in IEEE Transactions on Wireless Communications.

[12] J. Lehnert and M. Pursley, "Error probabilities for binary direct-sequence spread-spectrum communications with random signature sequences," IEEE Transactions on Communications, vol. 35, no. 1, pp. 87-98, Jan 1987.

[13] M. Lotfinezhad and B. Liang, "Effect of partially correlated data on clustering in wireless sensor networks," in Proc. IEEE International Conference on Sensor and Ad hoc Communications and Networks (SECON'04), Oct. 2004.

[14] A. Papoulis and S. U. Pillai, Probability, Random Variables, and Stochastic Processes, 4th ed. McGraw-Hill, 2002.

[15] S.G.Foss and S. Zuyev, "On a voronoi aggregative process related to a bivariate poisson process," Advances in Applied Probability, vol. 28, no. 4, pp. 965-981, 1996. 